\title{
Relationship between the induction of inflammatory processes and infectious diseases in patients with ischemic stroke
}

\author{
Marek Cieślak ${ }^{\boxplus}$, Andrzej Wojtczak² and Michał Cieślak³ \\ 1Nerology Department, Regional Polyclinical Hospital in Toruń, Toruń, Poland; 2Department of Crystal Chemistry and Biocrystallography, \\ Nicolaus Copernicus University in Toruń, Toruń, Poland; ${ }^{L}$ Ludwik Rydygier Collegium Medicum, Nicolaus Copernicus University in Bydgoszcz, \\ Bydgoszcz, Poland
}

Pro-inflammatory cytokines participate in the induction of ischemic stroke. So far, their participation in the cerebral ischemia was proven for the tumor necrosis factor TNF- $a$, interleukin-1 (IL-1), and interleukin-6 (IL-6). The release of the pro-inflammatory cytokines into the extracellular space causes the enlargement of the brain damage region, and consequently increases the neurological deficit and negatively affects the survival rate prognoses. That is confirmed by the increased concentration of proinflammatory cytokines in blood and the cerebrospinal fluid of patients with brain stroke, as well as by the research on the induced/experimental cerebral ischemia in animals. The pro-inflammatory cytokines participate in the migration of the reactive $\mathrm{T}$ lymphocytes to the regions of brain ischemia where they enhance the nerve tissue damage by down-regulation of microcirculation, induce the pro-thrombotic processes and release other neurotoxic cytokines. Also, in the early stage of cerebral ischemia, cytokines activate the axis hypothalamus-pituitary gland-adrenal cortex and increase the cortisol concentration in blood, what results in the decreased resistance to infectious diseases. Administration of the inhibitor of the interleukin-1 receptor (IL-1Ra) inhibits the inflammatory processes in the region of brain ischemia, and subsequently improves the prognosis for the size of the neurological deficit and the survival rate, as well as resistance to infectious diseases.

Key words: cytokines, interleukin-1 receptor, ischemic stroke

Received: 15 February, 2013; revised: 04 September, 2013; accepted: 12 September, 2013; available on-line: 18 September, 2013

\section{INDUCTION OF INFLAMMATORY PROCESSES IN THE CENTRAL NERVOUS SYSTEM IN THE COURSE OF ISCHEMIC STROKE}

During the ischemic stroke, the participation in the induction of inflammatory processes was proven for the pro-inflammatory cytokines, such as: tumor necrosis factor- $\alpha(\mathrm{TNF}-\alpha)$, interleukin (IL-1) (existing in two forms IL- $\alpha$ and IL- $\beta$ ), and interleukin-6 (IL- 6 ). Few reports indicate also the possible participation of IL-8 (chemokines, CXCL8) in ischemic stroke, which is produced in site of ischemia by the damaged tissue. In peripheral blood mononuclear cells (PBMC), the activity of IL- 8 mRNA is increased after the stroke. The serum concentration of IL- 8 increases after the stroke and remains elevated up to 1 month (Kostulas et al., 1998). The research has shown the increased concentration of the pro-inflammatory cytokines in blood and cerebrospi- nal fluid (Maas et al., 2009), and TNF- $\alpha$ in the region of the brain infarct (Sairanen et al., 2001; Dziewulska et al., 2003). In the explanation of that mechanism a significant contribution was provided by Polish scientists - Prof. Zaremba and Prof. Losy, who in 2001 and subsequent years, pointed to the significance of the proinflammatory cytokines for brain stroke (Zaremba et al., 2001; Zaremba et al., 2001; Zaremba et al., 2004). Concentration of these compounds in the intercellular space in the course of the cerebral ischemia increases by factor 40-60 (Lambertsen et al., 2005; Clausen et al., 2005; Clausen et al., 2008). Up to this date, there are only few reports on the role of anti-inflammatory cytokines in ischemic stroke. The published reports concern mainly the interleukin-10 (IL-10) (Basic Kes et al., 2008; Singh et al., 2013; Nayak et al., 2009). It is supposed that during the initial few hours the balance between the pro- and anti-inflammatory interleukins is disturbed due to the increased release of the pro-inflammatory cytokines and decreased release of IL-10. Researchers agree that concentration of this interleukin during initial hours of the stroke might be decreased (Singh et al., 2013; Nayak et al., 2012). Nayak A et al have proved the decrease of the IL-10 concentration in the serum during the initial $24 \mathrm{~h}$ of the stroke, although it has increased after 72 and 144 hours in patients who have clinically improved (Nayak et al., 2009). Simultaneously, in the early stages of the stroke, research showed the decrease in the concentration of the anti-inflammatory cytokine - IL-10, which is produced by leukocytes and macrophages originated from the blood monocytes (Basic Kes et al., 2008).

The activity of IL-6 is complex and still not fully known. The research on IL-6 has shown the correlation between the high concentration of that cytokine at early stages of the stroke (first 24 hours at the end of the first week) and the intensity of the infarct and the area of the brain infarct (Fassbender et al., 1994; Waje-Andreassen et al., 2005; Smith et al., 2004; Smith et al., 2004). Interleukin-6 reveals both pro- and antiinflammatory action (ambivalent effects). In stroke patients, concentration of IL-6 in blood is higher and is independent of the infarct volume, stroke severity, age, and risk factors for recurrent stroke (Smith et al., 2004; Smith et al., 2004; Whiteley et al., 2009). IL-6 can function as a pro-inflammatory cytokine by enhancing leukocyte recruitment by up-regulating the production of

e-mail: marcies@autograf.p

Abbreviations: BBB, blood-brain barrier; 3,6-DT, 3,6-dithiothalidomide; ICAM-1, intercellular adhesion molecule-1; IL, interleukin; TNF-a, tumor necrosis factor-a; PBMC, peripheral blood mononuclear cells; PWI-MRI, MR Perfusion Weighted Imaging; VCAM-1, vascular cell adhesion molecule-1. 
chemokines and adhesion molecule expression (Taga et al., 1997). IL-6 also serves as an anti-inflammatory cytokine by inhibiting the TNF- $\alpha$ expression and inducing the expression of soluble $\mathrm{TNF}-\alpha$ receptors and the IL-1R antagonist (Benveniste et al., 1995). It is known that the production of IL- 6 might be induced by different molecules, such as IL-1, TNF- $\alpha$, transforming growth factor-beta, and prostaglandins (PGs), as well as many other mediators such as $\beta$-amyloid, interferon- $\gamma$ $(\mathrm{IFN}-\gamma)$, and IL-4, which potentially might affect these complex processes. There are reports on the neuroprotective activity of IL-6, probably by enhanced expression and activity of the A1 adenosine receptors, as well as on the participation of that cytokine in the neurodegeneration process and neuron death (Biber et al., 2008; Pizzi et al., 2004; Nelson et al., 2004; Conroy et al., 2004). It is believed, that interleukin-6 plays an essential role in the post-stroke angiogenesis (Gertz et al., 2012). As early as within the first hours of the brain infarct, the increasing concentration of the pro-inflammatory cytokines induces inflammatory processes, which increase the infarct area, and consequently the degree of neurological deficit, what might have a prognostic significance. Cytokines present at every stage of cerebral stroke reveal the pro-inflammatory and pro-thrombotic activity. Increased expression of $\mathrm{TNF}-\alpha$ in the course of cerebral stroke activates the expression of the tissue factor and adhesive molecules for leukocytes, release of interleukin-1 (IL-1), nitrogen oxide, clotting factor VIII (von Willebrand factor), the factor activating blood thrombocytes and endothelin, but suppresses the thrombomodulin-protein C-protein S system, decreases the concentration of the tissue-plasminogen activator and release of the plasminogen activator inhibitor-1 (Tuttolomondo et al., 2008).

Research of Tarkowski et al. on patients suffering from cerebral ischemic stroke has revealed the presence of TNF- $\alpha$ in the cerebrospinal fluid even 3 months after falling sick (Tarkowski et al., 1997). Zaremba and Losy have confirmed the increased concentration of TNF- $\alpha$ in the cerebrospinal fluid during the initial 24 hours of the stroke, and have shown the correlation between this concentration and the infarct intensity. Results of the lately finished research have revealed the correlation between the concentration of IL- 6 in the cerebrospinal fluid within the initial 6 hours of the cerebral ischemia and the size of the brain infarct determined in the 24th hour (Beridze et al., 2011). In 1995, Tarkowski et al. have found the increased concentration of IL-1 $\beta$ in the majority of the examined patients with brain stroke, which was also observed by other authors even in small brain infarct (Sun et al., 2009).

The research on the level of cytokines in blood does not provide such unequivocal results, and the reports are contradictory. Moreover, analyses of cytokines in the peripheral blood might but do not have to reflect the synthesis of cytokines in the central nervous system. In particular, the controversies focus on TNF- $\alpha$. There are some reports not revealing the increase of the concentration of that cytokine after brain stroke (Basic Kes et al., 2008). Montaner et al. have not revealed any significant elevation of the concentration of that cytokine in the blood serum after brain stroke during the initial 24 hours, although a correlation was shown between the concentration of that compound and changes in perfusion in PWI-MRI (MR Perfusion Weighted Imaging) (Montaner et al., 2003). Although the role of interleukin- $1 \beta$ in animals in the experimental stroke is proven, there are no observations of the significant increase of its concentration in patients with

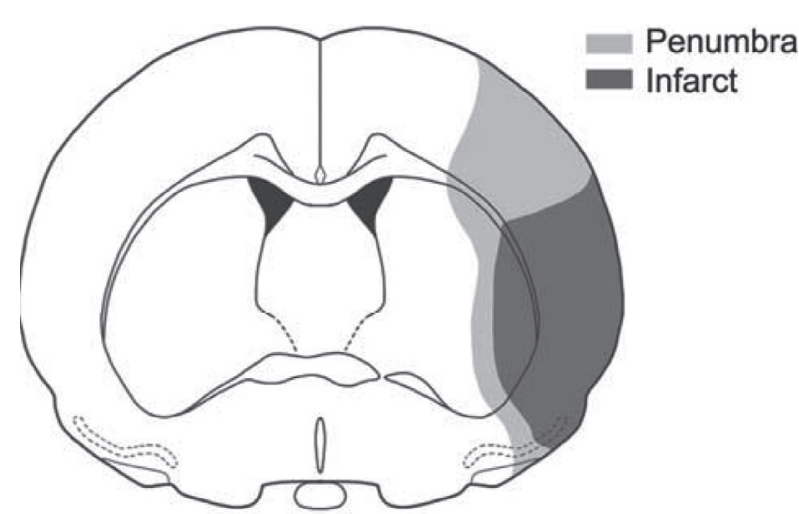

Figure 1. Schematic illustration of penumbra and infract localization and grade of the infection in the course of the stroke (modified from Lambertsen et al., 2005).

brain infarct (Tarkowski et al., 1995; Emsley et al., 2007; Astrup et al., 1981).

Many important results are obtained from research on animals. The animal model of ischemic stroke can be obtained by inducing the obliteration of the middle cerebral artery (Rodriguez-Mercado et al., 2012). In humans, the sudden occlusion of the middle cerebral artery causes clinically severe neurological syndrome with the contralateral facial weakness, severe hemiparesis and hemisensory loss, homonymus hemianopia, global aphasia (dominant hemisphere) usually with cerebral edema manifested by the consciousness disturbances. In the experimental brain infarct in animals, the increased concentration of TNF- $\alpha$ and IL- 1 is observed during the initial hours of ischemia, with the maximum reached between 12 and 24 hours, and remains elevated for few more days (Nayak et al., 2009; Fassbender et al., 1994; Smith et al., 2004; Smith et al., 2004). Few reports on IL-6 have shown that in rats mRNA of that cytokine was significantly elevated already after 3 hours of infarct and remained elevated for 24 hours.

After severe cerebral ischemia (initial 7 days), a period of convalescence occurs, with the possible influence of cytokine IL-6 on angiogenesis (Gertz et al., 2012). In small strokes (lacunar stroke), no significant elevation of concentration of pro-inflammatory cytokines in blood was found, what might be related to the lack of damage of the blood-brain barrier (BBB) (Licata et al., 2009).

The effect of these cytokines on the evolution of the brain infarct depends on their increasing concentration in the peri-infarct region (penumbra) at the early stage of brain ischemia, what corresponds to the therapeutic window (less than $4.5 \mathrm{~h}$ ), and that was found in humans and in animals in the experimental brain stroke. The peri-infarct region (penumbra) is defined as a region directly surrounding the stroke core, in which the blood flow is lowered and the activity of the neurons is impaired, but the structural integrity of cells is maintained (Astrup et al., 1981). During the stroke evolution, the dynamically changing penumbra might either return to the regions of brain with the proper functioning or become a region of the complete ischemic stroke. TNF- $\alpha$ and interleukin 1- $\beta$ are the first cytokines released before the manifestation of the inflammation symptoms and induce the synthesis of subsequent pro-inflammatory cytokines such as IL-6 or chemokines. Research on animals has shown that in penumbra, TNF- $\alpha$ and IL-1 are synthesized by microglia cells, intrathecal macrophages and migrating macro- 
phages (originated from the blood monocytes), but IL-6 is synthesized by microglia cells and neurons (Lambertsen et al., 2005; Clausen et al., 2008).

In the development of inflammatory processes in the central nervous system, an important role is played by the reactive lymphocytes $\mathrm{T}$, migrating through the endothelium to the region of ischemia (Becker et al., 2001; Becker et al., 1998). Among the pro-inflammatory cytokines, a special role is played by interleukin- $1 \beta$, which participates in the activation of the synthesis of IL- 2 and induces the expression of its receptor. Moreover, IL-1 $\beta$ stimulates the synthesis of IFN- $\gamma$ and IL- 6 by lymphocytes, macrophages, and endothelial cells and enhances the adhesion of lymphocytes $\mathrm{T}$ to the endothelial cells. Pro-inflammatory cytokines induce the expression of such molecules as selectin E, intercellular adhesion molecule-1 (ICAM-1), and vascular cell adhesion molecule-1 (VCAM-1), as well as the activation of leucocytes. The adhesion proteins located on the surface of the endothelial cells, such as VCAM-1, participate in the penetration of the reactive lymphocytes $\mathrm{T}$ into $\mathrm{CNS}$, since they are bound to the $\alpha 4 \beta 1$ integrin (VLA-4) located on the lymphocyte surface. Leucocytes appearing in the regions of the cerebral ischemia enhance damage of the nervous tissue, since they adhere to endothelium, what impairs the brain blood flow. The lymphocytes activated in the regions of the brain ischemia release subsequent neurotoxic cytokines, proteolytic enzymes and compounds contracting the vessels, and induce the expression of pro-clotting factors in endothelial cells. These processes intensify the local brain ischemia (Becker et al., 2001; Becker et al., 1998). Pro-inflammatory cytokines released during brain ischemia cause the lesion of the blood-brain barrier, what results in the exposition of some antigens of the central nervous system to the activity of the peripheral immune system, which recognizes these antigens (eg. S100 protein) as the strange ones and induces the inflammatory response in the peripheral blood with cytokines produced by leucocytes (Becker et al., 2001; Becker et al., 1998).

\section{Perspectives of new anti-inflammatory therapies of cerebral ischemic stroke}

In ischemic stroke, $\mathrm{TNF}-\alpha$ plays a crucial role in brain damage. In a mouse model of focal ischemic stroke, in cultured cells, an analog of thalidomide - 3,6-dithiothalidomide $(3,6-\mathrm{DT})$ is a selective inhibitor of TNF- $\alpha$ synthesis, suggesting that 3,6-DT has an anti-inflammatory effect (Yoon et al., 2013). Administration of 3,6'-DT within the first 3 hours after the stroke onset reduced the infarct volume, neuronal death, and neurological deficits. Thalidomide was effective only when administered prior to the stroke. Neuroprotection was accompanied by a decreased inflammation; 3,6'-DT-treated mice exhibited the reduced expression of $\mathrm{TNF}-\alpha$, interleukin- $1 \beta$, and inducible nitric oxide synthase; reduced numbers of activated microglia/macrophages, astrocytes, and neutrophils; and reduced expression of intercellular adhesion molecule-1 in the ischemic brain tissue. The 3,6'DT treatment attenuated stroke-induced disruption of the blood-brain barrier by a mechanism that appears to involve suppression of matrix metalloproteinase-9 and preservation of occludin. Treatment with 3,6'-DT did not reduce the ischemic brain damage in mice lacking $\mathrm{TNF}-\alpha$ receptors, consistent with a critical role for suppression of $\mathrm{TNF}-\alpha$ production and $\mathrm{TNF}-\alpha$ signaling in the therapeutic action of 3,6'-DT. Already within the initial hours of cerebral ischemic stroke, the increase of the concentration of pro-inflammatory cytokines in serum, including IL-6 occurs. Increase in the concentration of IL-6 correlates with the area and severity of the stroke and its clinical outcome (Basic Kes et al., 2008, Switzer et al., 2012). Results of Switzer and coworkers (Switzer et al., 2012) suggest that the administration of minocycline in the acute stage of the stroke decreases the IL-6 concentration. Minocycline suppresses the activation of neutrophils, macrophages and microglia cells. Since the research of Switzer et al. (Switzer et al., 2012) has not been randomized, it should be continued.

\section{MECHANISM OF DECREASED PERIPHERAL IMMUNITY IN PATIENTS WITH ISCHEMIC STROKE}

Infectious diseases in patients suffering from cerebral ischemic stroke occur more frequently than in healthy humans, and constitute a frequent complication of the main disease. That is influenced by different external factors, such as patient immobilization, bronchial toilet, catheterization of the urinary bladder and other. Among these factors are also: pneumonia, inflammation of the urinary tracts, and skin inflammation. Nowadays it is known that in this group of patients a decrease of the peripheral immunity is observed, what results in an increased susceptibility to infectious diseases. The reason leading to such effect is the increase in the concentration of the pro-inflammatory cytokines in the central nervous system and in blood in the course of cerebral ischemic stroke.

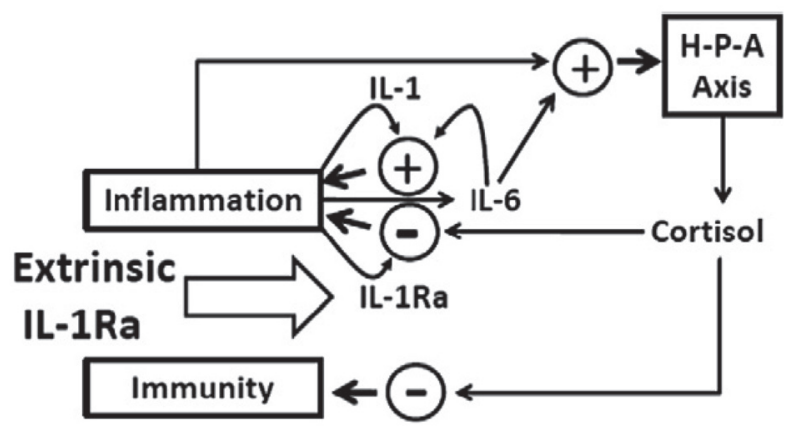

Figure 2. Schematic illustration of interactions between IL-1 blockade and the HPA axis, and their relationships with inflammation and immunity (according to Smith et al. (2004).

In 2001 the research of Boutin and coworkers (Boutin et al., 2001) conducted on mice revealed that the administration of the recombinant antagonist of the IL-1 receptor into the ventricular system caused the significant decrease of the area of the brain infarct. In 2005, the research by Emsley et al. revealed a decrease of the markers of the biological activity such as the amount of neutrophiles and leucocytes in the peripheral blood and a decrease of the concentration of C-reactive protein (CRP) and IL-6 in patients treated with the inhibitor of the IL-1 receptor (rhIL-1Ra) (Emsley et al., 2005). After 3 months from the date of the IL-1Ra administration, patients with cortical stroke were in better neurological state than those receiving placebo. The administration of IL-1Ra either peripherally or into the ventricular system, significantly decreased the neuronal damage in transient or chronic occlusion, of the middle cerebral artery, complete brain ischemia, intraperitoneal hypoxia/ischemia, traumatic excytotoxicity, hemorrhage, or heart stroke. The best neuroprotective effect was achieved when IL- 
$1 \mathrm{Ra}$ was administered during the initial 3 hours of occlusion of the middle cerebral artery occurrence, 8-12 hours for complete brain ischemia and the brain injury. Administration of IL-1Ra caused a decrease of the area of brain damage, better neuron survival rate, decrease of the cerebral edema and invasiveness of the peripheral immunological cells.

Current results of Smith and coworkers (Smith et al., 2005) suggest that at the early stage of cerebral ischemic stroke, there is an increase in the concentration of IL-1, IL-6, C-reactive protein (CRP) and activation of the axis hypothalamus-pituitary gland-adrenal cortex (Ormstad et al., 2011). High blood concentration of CRP and IL-6 correlates with the extensiveness of the brain damage region and neurological symptoms (Smith et al., 2005; Ormstad et al., 2011). The authors have shown that activation of the axis hypothalamus-pituitary gland-adrenal cortex co-exists with the increase of the cortisol concentration in blood, what seems to be the reason for decreased immunity (Emsley et al., 2008). That process accompanies the increased production of IL-1 and TNF- $\alpha$. Since IL-1 is strongly involved in pathophysiology of the cerebral ischemia and affects the congenital immunity, Smith et al. (2005) have conducted their research with the exogenous administration of inhibitor of the IL-1 receptor (IL-1Ra) in the experimental cerebral ischemic stroke. Administration of IL-1Ra resulted in the significant decrease of the brain damage region in the cerebral ischemia, correlated with a decrease of the cortisol concentration in blood, what seems to be related to the reversibility in the immunity suppression. Currently, the research is conducted (II phase) in patients with ischemic stroke, who are intravenously treated with IL-1Ra. Unfortunately, so far the effect of IL-1Ra on the leukocyte-dependent peripheral immunity remains unknown. Research by the mentioned authors brings hope that in the future there will be a chance of treating patients with cerebral ischemic stroke by means of the administration of the IL-1 receptor inhibitor, and consequently a decrease of the neurological deficit, improved prognosis as to survival, and a decrease of incidences of infectious diseases.

\section{REFERENCES}

Astrup J, Siesjö BK, Symon L (1981) Thresholds in cerebral ischemia the ischemic penumbra. Stroke 12: 723-725.

Basic Kes VB, Simundic AM, Nicolac N et al. (2008) Pro-inflammatory and anti-inflammatory cytokines in acute ischemic stroke and their relation to early neurological deficit and stroke outcome. Clinical Biochemistry 41: 1330-1334.

Becker KJ (1998) Inflammation and acute stroke. Curr Opin Neurol 11: 45-49.

Becker KJ (2001) Targeting the central nervous system inflammatory response in ischemic stroke. Curr Opin Neurol 14: 349-353.

Benveniste E, Tang L, Law R (1995) Differential regulation of astrocyte TNF-alpha expression by the cytokines TGF-beta, IL-6 and IL-10. Int J Dev Neurosci 13: 341-349.

Beridze M, Sanikidze T, Shakarishvili R et al. (2011) Selected acute phase CSF factors in ischemic stroke: findings and prognostic value. BMC Neurol 30: 11-41.

Biber K, Pinto-Duarte A, Wittendrop MC (2008) Interleukin-6 upregulates neuronal adenosine A1 receptors: implications for neuromodulation and neuroprotection. Neuropsychopharmacology 33: 2237-2250.

Boutin H, LeFeuvre RA, Horai R et al. (2001) Role of IL-1alpha and IL-1beta in ischemic brain damage. J Neurosci 1: 5528-5534.

Clausen BH, Lambertsen KL, Babcock AA et al. (2008) Interleukin1 beta and tumor necrosis factor-alpha are expressed by different subsets of microglia and macrophages after ischemic stroke in mice. J Neuroinflammation 5: 46.

Clausen BH, Lambertsen KL, Meldgaard M et al. (2005) Quantitative in situ hybridization and polymerase chain reaction study of microglialmacrophage expression of interleukin-1beta mRNA following per- manent middle cerebral artery occlusion in mice. Neuroscience 132: 879-892.

Conroy SM, Nguyen V, Quina LA et al. (2004) Interleukin-6 produces neuronal loss in developing cerebellar granule neuron cultures. $J$ Neuroimmunol 155: 43-54.

Dziewulska D, Mossakowski MJ (2003) Cellular expression of tumor necrosis factor a and its receptors in human ischemic stroke. Clin Neuropathol 22: 35-40.

Emsley HC, Smith CJ, Gavin CM et al. (2007) Clinical outcome following acute ischemic stroke relates to both activation and autoregulatory inhibition of cytokine production. BMC Neurol 28: 7: 5 .

Emsley HC, Smith CJ, Georgiou RF, Vail A, Hopkins SJ, Rothwell NJ et al. (2005) A randomised phase II study of interleukin-1 receptor antagonist in acute stroke patients. J Neurol Neurosurg Psychiatry 76: 1366-1372.

Emsley HC, Smith CJ, Tyrrell PJ et al. (2008) Inflammation in acute ischemic stroke and its relevance to stroke critical care. Neurocrit Care 9: 125-138.

Fassbender K, Rossol S, Kammer T et al. (1994) Proinflammatory cytokines in serum of patients with acute cerebral ischemia: kinetics of secretion and relation to the extent of brain damage and outcome of disease. J Neurol Sci 122: 135-139.

Gertz K, Kronenberg G, Kälin RE, Baldinger T, Werner C, Balkaya M, Eom GD, Hellmann-Regen J, Kröber J, Miller KR, Lindauer U, Laufs U, Dirnagl U, Heppner FL, Endres M (2012) Essential role of interleukin-6 in post-stroke angiogenesis. Brain 135: 1964-1980.

Kostulas N, Kivisäkk P, Huang Y, Matusevicius D, Kostulas V, Link $\mathrm{H}$ (1998) Ischemic stroke is associated with a systemic increase of blood mononuclear cells expressing interleukin-8 mRNA. Stroke 29: 462-466.

Lambertsen KL, Meldgaard M, Ladeby R et al. (2005) A quantitative study of microglial-macrophage synthesis of tumor necrosis factor during acute and late focal cerebral ischemia in mice. J Cereb Blood Flow Metab 25: 119-135.

Licata G, Tuttolomondo A, Di Raimondo D, Corrao S, Di Sciacca R, Pinto A (2009) Immuno-inflammatory activation in acute cardioembolic strokes in comparison with other subtypes of ischaemic stroke. Thromb Haemost 101: 929-937.

Maas MB, Furie KL (2009) Molecular biomarkers in stroke diagnosis and prognosis. Biomark Med 3: 363-383.

Montaner J, Rovira A, Molina CA et al. (2003) Plasmatic level of neuroinflammatory markers predict the extent of diffusion-weighted image lesions in hyperacute stroke. J Cereb Blood Flow Metab 23: 1403-1407.

Nayak A, Kashyap R, Kabra D, Purohit H, Taori G, Daginawala H (2012) Time course of inflammatory cytokines in acute ischemic stroke patients and their relation to inter-alfa trypsin inhibitor heavy chain 4 and outcome. Ann Indian Acad Neurol 15: 181-185.

Nayak A, Kashyap R, Purohit H, Kabra D, Taori G, Daginawala H (2009) Evaluation of the inflammatory response in sera from acute ischemic stroke patients by measurement of IL-2 and IL-10. Inflamm Res 58: 687-691.

Nelson TE, Netzeband JG, Gruol DL (2004) Chronic interleukin-6 exposure alters metabotropic glutamate receptor-activated calcium signalling in cerebellar Purkinje neurons. Eur J Neurosci 20: 2387-400.

Ormstad H, Aass HC, Lund-Sørensen N et al. (2011) Serum levels of cytokines and C-reactive protein in acute ischemic stroke patients, and their relationship to stroke lateralization, type, and infarct volume. I Neurol 258: 677-685.

Pizzi M, Sarnico I, Boroni F et al. (2004) Prevention of neuron and oligodendrocyte degeneration by interleukin-6 (IL-6) and IL-6 receptor/IL-6 fusion protein in organotypic hippocampal slices. Mol Cell Neurosic 25: 301-311.

Rodriguez-Mercado R, Ford GD, Xu Z, Kraiselburd EN, Martinez MI, Eterović VA, Colon E, Rodriguez IV, Portilla P, Ferchmin PA, Gierbolini L, Rodriguez-Carrasquillo M, Powell MD, Pulliam JV, McCraw CO, Gates A, Ford BD (2012) Acute neuronal injury and blood genomic profiles in a nonhuman primate model for ischemic stroke. Comp Med 62: 427-438.

Sairanen T, Carpen O, Karjalainen-Lindsberg ML et al. (2001) Evolution of cerebral tumor necrosis factor-alpha production during human ischemic stroke. Stroke 32: 1750-1758.

Singh HV, Pandey A, Shrivastava AK, Raizada A, Singh SK, Singh N (2013) Prognostic value of neuron specific enolase and IL-10 in ischemic stroke and its correlation with degree of neurological deficit. Clin Chim Acta 18: 419: 136-138.

Smith C, Emsley H, Udeh Ch et al. (2012) Interleukin-1 receptor antagonist reverses stroke-associated peripheral immune suppression. Cytokine 58: 384-389.

Smith CJ, Emsley HC, Gavin CM, Georgiou RF, Vail A, Barberan EM, del Zoppo GJ, Hallenbeck JM, Rothwell NJ, Hopkins SJ, Tyrrell PJ (2004) Peak plasma interleukin-6 and other peripheral markers of inflammation in the first week of ischaemic stroke correlate with brain infarct volume, stroke severity and long-term outcome. BMC Neurol 15: 4: 2. 
Sun Y, Lu CJ, Lin CH, Wen LL (2009) Interleukin-1beta is increased in the cerebrospinal fluid of patients with small infarcts. Eur J Neurol 16: 858-863.

Switzer J, Sikora A, Ergul A, Waller JL, Hess DC, Fagan SC (2012) Minocycline prevents IL-6 increase after acute ischemic stroke. Transl Stroke Res 3: 363-368.

Taga T, Kishimoto T (1997) Gp130 and the interleukin-6 family of cytokines. Annu Rev Immunol 15: 797-819.

Tarkowski E, Rosengren L, Blomstrand C, Wikkelso C, Jensen C, Ekholm S, Tarkowski A (1997) Intrathecal release of pro- and anti-inflammatory cytokines Turing stroke. Clin Exp Immunol 110: 492499.

Tarkowski E, Rosengren L, Blomstrand Ch et al. (1995) Early intrathecal production of interleukin-6 predicts the size of brain lesion in stroke stroke. 26: 1393-1398.

Tuttolomondo A, Di Raimondo D, di Sciacca R et al. (2008) Inflammatory cytokines in acute ischemic stroke. Curr Pharm Des 14: 3574 3589.

Waje-Andreassen U, Kråkenes J, Ulvestad E et al. (2005) IL-6: an early marker for outcome in acute ischemic stroke. Acta Neurol Scand 111: 360-365.
Whiteley W, Jackson C, Lewis S, Lowe G, Rumley A, Sandercock P, Wardlaw J, Dennis M, Sudlow C (2009) Inflammatory markers and poor outcome after stroke: a prospective cohort study and systematic review of interleukin-6. PLoS Med 6: 1-11.

Yoon J, Lee J, Tweedie D, Mughal M, Chigurupati S, Greig N, Mattson M (2013) 3,6'-dithiothalidomide improves experimental stroke outcome by suppressing neuroinflammation. I Neurosci Res 91: 671680.

Zaremba J, Losy J (2001) Early TNF-alpha levels correlate with ischaemic stroke severity. Acta Neurol Scand 104: 288-295.

Zaremba J, Skrobanski P, Losy J (2001) Tumour necrosis factor-alpha is increased in the cerebrospinal fluid and serum of ischaemic stroke patients and correlates with the volume of evolving brain infarct. Biomed Pharmacother 55: 258-263.

Zaremba J, Losy J (2004) Cytokiny w klinicznym i doświadczalnym udarze niedokrwiennym mózgu. Neurologia $i$ Neurochirurgia Polska 38 (Suppl. 1): 57-62 (in Polish). 